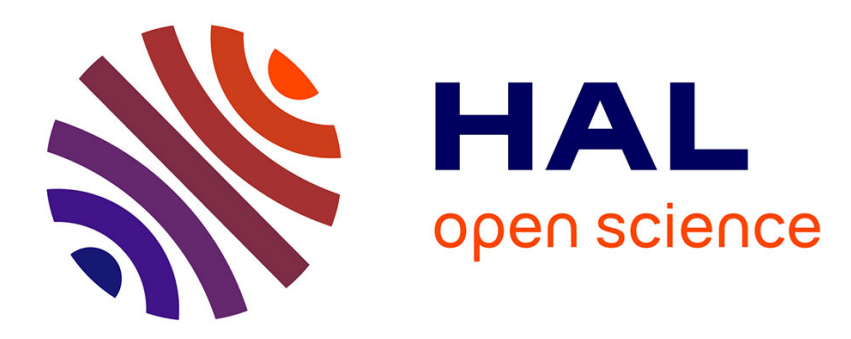

\title{
Modeling of plane Couette flow. II. On the spreading of a turbulent spot
}

\author{
Maher Lagha
}

\section{To cite this version:}

Maher Lagha. Modeling of plane Couette flow. II. On the spreading of a turbulent spot. Physics of Fluids, 2007, 19 (10), pp.104108. 10.1063/1.2793143 . hal-01023089

\section{HAL Id: hal-01023089}

https://hal-polytechnique.archives-ouvertes.fr/hal-01023089

Submitted on 21 Jul 2014

HAL is a multi-disciplinary open access archive for the deposit and dissemination of scientific research documents, whether they are published or not. The documents may come from teaching and research institutions in France or abroad, or from public or private research centers.
L'archive ouverte pluridisciplinaire HAL, est destinée au dépôt et à la diffusion de documents scientifiques de niveau recherche, publiés ou non, émanant des établissements d'enseignement et de recherche français ou étrangers, des laboratoires publics ou privés. 


\section{AIP | Physics of Fluids}

\section{Modeling of plane Couette flow. II. On the spreading of a turbulent spot}

Maher Lagha

Citation: Physics of Fluids (1994-present) 19, 104108 (2007); doi: 10.1063/1.2793143

View online: http://dx.doi.org/10.1063/1.2793143

View Table of Contents: http://scitation.aip.org/content/aip/journal/pof2/19/10?ver=pdfcov

Published by the AIP Publishing

Articles you may be interested in

Numerical simulation of bubble dispersion in turbulent Taylor-Couette flow

Phys. Fluids 26, 043304 (2014); 10.1063/1.4871728

Bi-stability in turbulent, rotating spherical Couette flow

Phys. Fluids 23, 065104 (2011); 10.1063/1.3593465

Transient growth and minimal defects: Two possible initial paths of transition to turbulence in plane shear flows Phys. Fluids 16, 3515 (2004); 10.1063/1.1775194

Interaction between Ekman pumping and the centrifugal instability in Taylor-Couette flow

Phys. Fluids 15, 467 (2003); 10.1063/1.1534108

On a self-sustaining process in shear flows

Phys. Fluids 9, 883 (1997); 10.1063/1.869185

\section{A|P| Journal of}

Journal of Applied Physics is pleased to announce André Anders as its new Editor-in-Chief 


\title{
Modeling of plane Couette flow. II. On the spreading of a turbulent spot
}

\author{
Maher Lagha ${ }^{\text {a) }}$ \\ Laboratoire d'Hydrodynamique (LadHyX), École Polytechnique, F-91128 Palaiseau, France
}

(Received 23 March 2007; accepted 11 July 2007; published online 29 October 2007)

\begin{abstract}
In this paper, we study the spreading mechanism of turbulent spots in plane Couette flow, where fluid is sheared between two parallel plates moving in opposite directions. The analysis of the coherent structures on the border between the laminar and turbulent regions reveals the existence of many vortices with wall-normal axes occupying the full gap between the plates. The streamwise component of the velocity field of these vortices is the streaks. Due to their self-advection, these vortices move parallel to the plates. During their motions, they carry the other perturbation components such as the streamwise and spanwise vortices. (c) 2007 American Institute of Physics.
\end{abstract}

[DOI: $10.1063 / 1.2793143$ ]

\section{INTRODUCTION}

Plane Couette flow (PCF), shear flow between two parallel plates moving in opposite directions with velocities $\pm U_{p}$, experiences a transition to turbulence marked by the nucleation and growth of turbulent spots, i.e., patches of turbulent flow scattered amid laminar flow and separated from it by well-defined fronts (e.g., Ref. 1).

This transition is not restricted to the PCF case but also occurs in other shear flows with great practical interest, such as plane Poiseuille ${ }^{2}$ and boundary layer flows. ${ }^{3}$ Despite a large body of numerical ${ }^{4-6}$ and laboratory ${ }^{7-10}$ experiments, many questions regarding such transition remain unanswered, such as the mechanisms involved in the growth of turbulent spots. ${ }^{11,12}$

Based on experiments in boundary layer flow, Gad-ElHak et $a l^{3}$ proposed a mechanism called growth by destabilization. The spot was observed to travel with a lower velocity than the surrounding flow. Hence it acts as a blockage, and the laminar flow field on the outskirts of this spot is accelerated. The base flow as well as its linear stability property are modified and the growth of infinitesimal perturbations occurs.

Dauchot and Daviaud ${ }^{1}$ discussed this mechanism in an experimental study of the PCF. Externally applied perturbations that trigger turbulent spots were made by injecting turbulent jets into the laminar flow. They found velocity profiles indicating that the flow is accelerated outside the spot, supporting the mechanism of Ref. 3. But a direct demonstration of this mechanism has not yet appeared.

Tillmark $^{8}$ experimentally analyzed the flow field in the vicinity of the spot in the PCF. He found that, in the spanwise direction, the spot forces the fluid outwards, giving rise to a spanwise outflow filling all of the gap between the two plates. The flow outside the spot is hence modified and he suggested that the spanwise growth of turbulent spots can be due to the destabilization mechanism of Gad-El-Hak et al. $^{3}$ In the numerical study of Schumacher and Eckhardt, ${ }^{5}$ a more complete picture of the flow on the outskirts of the spot was

\footnotetext{
a)Electronic mail: maher@ladhyx.polytechnique.fr
}

given. In addition to the spanwise outflow observed in Ref. 8, they found a streamwise inflow toward the spot. They argued that the flow outside the spot plays an important role in the spreading of the spot. They stressed the fact, however, that this spreading is driven by a nonlinear mechanism.

In their experimental investigations of the dynamics of spots in the plane Poiseuille flow, Carlson et al. ${ }^{2}$ noted that the spots were accompanied by oblique waves at their leading edge (wing tips). It was difficult, however, to find out whether the waves broke down and formed the new turbulence on the wing tips, or whether they are overtaken by the existing turbulence.

The nature of these waves and their role in the spreading of the spot were studied, using numerical simulations, by Henningson et al. ${ }^{13}$ Due to the modification of its stability properties by the presence of the spot, the surrounding flow is susceptible to unstable oblique Tollmien-Schlichting waves, which may grow and then break down into turbulence. However, the linear growth rate of these waves calculated by Henningson ${ }^{14}$ is too small compared to the observed one. Therefore, he suggested that the waves attain their large growth rate by some additional mechanisms.

Furthermore, Alavyoon et al. ${ }^{15}$ compared spots in plane Poiseuille and boundary layer flows and pointed out the absence of waves at the wing tips of spots in the latter case. According to these authors, this indicates that if the same spreading mechanism is at work in both cases, then the waves are of no importance for the spreading itself, whereas if these waves play an important role in the spreading of spots in plane Poiseuille flow, then the spreading mechanisms are different and depend on the flow configurations. Hence, the role of the waves in the breakdown process and in the spreading mechanism of the turbulent domain remains unclear and needs further study, as noted by Henningson.

Therefore, the question of which mechanism is involved in the spreading of spots in shear flows is to a large extent open, and despite a large body of experiments, a simple intuitive physical picture has been lacking.

An attempt to tackle such a question led us to derive a model for PCF, presented in Ref. 16. The outline of this paper is as follows. In Sec. II, the model is introduced and 
some numerical results on the dynamics of turbulent spots are described. Then the structure of the flow at the boundary between laminar and turbulent domains is analyzed and the spreading mechanism is elucidated in Sec. III. This mechanism is further illustrated using a simple one-dimensional model. The main results of this paper are assessed in Sec. IV.

\section{GROWTH OF A TURBULENT SPOT}

\section{A. The model}

In Part I (Ref. 16), a model for the transitional plane Couette flow has been derived from the Navier-Stokes equations using the Galerkin method. First, lengths were scaled by the half-gap between the plates $h$, and velocities by $U_{\mathrm{p}}$. Second, the streamwise $(x)$, wall-normal $(y)$, and spanwise $(z)$ velocity components were expanded, respectively, as

$$
\begin{aligned}
& u(x, z, t, y)=U(y)+U_{0}(x, z, t) R_{0}(y)+U_{1}(x, z, t) R_{1}(y), \\
& v(x, z, t, y)=V_{1}(x, z, t) S_{1}(y), \\
& w(x, z, t, y)=W_{0}(x, z, t) R_{0}(y)+W_{1}(x, z, t) R_{1}(y),
\end{aligned}
$$

where $U(y)=U_{b} y$ for $y \in[-1,1]$ is the dimensionless base flow $\left(U_{b}=1\right)$ and the perturbation components are $U_{0}, W_{0}$, $U_{1}, W_{1}$, and $V_{1}$. The $y$-dependent functions satisfy the no-slip boundary conditions on the plates $y= \pm 1$ and are polynomials, $\quad R_{0}(y)=B\left(1-y^{2}\right), \quad R_{1}(y)=C y\left(1-y^{2}\right), \quad$ and $\quad S_{1}(y)=A(1$ $\left.-y^{2}\right)^{2}$, where $A, B$, and $C$ are constants. The model consists of a set of three partial differential equations governing the stream functions $\Psi_{0}$ and $\Psi_{1}$ and the velocity potential $\Phi_{1}$ of the velocity components $U_{0}, W_{0}, U_{1}, V_{1}, W_{1}$, and are given in the Appendix. The control parameter is the Reynolds number $R=U_{p} h / \nu$, where $\nu$ is the kinematic viscosity.

The derivation of models truncated at higher orders is possible, however we will have to settle with the present model. In fact, the use of such a low-dimensional model can be justified by some features of the PCF. First, the considered Reynolds numbers are close to the stability threshold $R_{g}$ $\sim 175$ (Ref. 16). For such numbers, the turbulent structures are observed to fill the entire gap between the plates (see, e.g., Ref. 10). Second, the correction to the laminar profile is already contained in the model and is represented by the streamwise velocity component $U_{1} R_{1}$. This component was shown to be important in the generation of the quadrupolar large-scale flow. ${ }^{16}$

Some prerequisites in relation to the previous part (Ref. 16) are now introduced. The wall-normal velocity associated with the streamwise vortices is represented by $V_{1}$. This velocity induces the streaks $U_{0}$ through the lift-up mechanism. In the half-space $y \geqslant 0$, regions where $U_{0}$ is positive (negative) correspond to high (low) speed streaks. In the other half-space $y \leqslant 0$, the situation is reversed. Then, to an $x$-dependent streak, $U_{0}$ corresponds to a spanwise velocity component $W_{0}$ so that the two-dimensional flow $\left(U_{0}, W_{0}\right)$ satisfies the continuity equation $\partial_{x} U_{0}+\partial_{z} W_{0}=0$. Since this flow has a Poiseuille-like cross-stream profile $R_{0}(y)$, it is termed drift-flow herein.

\section{B. Coherent structures on the front}

A standard Fourier pseudospectral method with periodic boundary conditions in the streamwise and spanwise directions has been implemented for the integration of the equations of the model (A4)-(A6). A second-order AdamsBashforth scheme is used for the advancement in time. Simulations were performed in a domain of size $\left(L_{x} \times L_{z}\right)$ $=(32 \times 32)$ with space steps $\delta x=\delta z=0.125$ and $\delta t=0.001$. With this resolution, small-scale in-plane structures such as streaks and streamwise vortices with a streamwise length about $4 h$ and a spanwise extent about $2 h$ are resolved with 16 collocation points in the spanwise direction and 32 in the streamwise direction. Regarding the time increment, smaller time steps did not produce results different from those shown here during comparable time lengths.

As an initial condition, we take localized functions $\Psi_{0}$, $\Psi_{1}$, and $\Phi_{1}$,

$$
\begin{aligned}
\Psi_{0}(x, z, t=0) & =\Psi_{1}(x, z, t=0) \\
& =\Phi_{1}(x, z, t=0) \\
& =A \exp ^{-\left(x^{2}+z^{2}\right) / \sigma},
\end{aligned}
$$

where $A$ is an amplitude and $\sigma$ is related to the size of the initial turbulent domain. ${ }^{16}$

Tracking the growth of a turbulent spot can be done by using one component of the velocity or vorticity fields at a given $y$ plane. Of particular benefit for our present study is the wall-normal vorticity associated to the drift flow $\left(U_{0}, W_{0}\right)$. Figure 1 displays gray-level snapshots of $\Delta_{2} \Psi_{0}$ $=\partial_{x} W_{0}-\partial_{z} U_{0}$, where $\Delta_{2}=\partial_{x x}+\partial_{z z}$, at different times after initiation. The spot grows and contaminates the laminar domain at $t \sim 210$.

Flow structures at the boundaries between the laminar and turbulent domains are the elements needed for understanding the spreading mechanism of the spots. Arrows in Fig. 1 (at $t=156$ ) show two adjacent patches with opposite signed vorticity $\Delta_{2} \Psi_{0}$ lying on the front propagating to the right. As shown in Fig. 2, these patches correspond to two counter-rotating vortices $\left(U_{0}, W_{0}\right)$. First, streamwise streaks $U_{0}$ are easily identified as regions where $\left|U_{0}\right| \gg\left|W_{0}\right|$. The sign of $U_{0}$ is alternating in the spanwise direction between positive and negative values, so that when the centers of the vortices are aligned along the $z$ direction, the distance between the centers corresponds roughly to the width of the streak. This distance varies from $1 h$ to $3 h$, as can be seen from Fig. 2. Second, as may be inferred from the sense of rotation of both vortices, this dipole is propagating to the right. Before studying the origin and the consequence of this motion, it is instructive to track the expansion of the turbulent spot with the remaining flow velocity components.

Figure 3 displays the spatial distribution of the wallnormal velocity $V_{1}$ and the flow field $\left(U_{1}, W_{1}\right)$, corresponding to the region in Fig. 2. The reconstruction of the total flow field $\left(\left(U_{1}, W_{1}\right) R_{1}(y), V_{1} S_{1}(y)\right)$ in this region reveals a crescent vortex. Its legs are two streamwise vortices that regenerate the streaks $U_{0}$ through the lift-up effect. There are two kinds of crescent vortices. During the spreading of the spot, both kinds are present inside the turbulent domain, but 

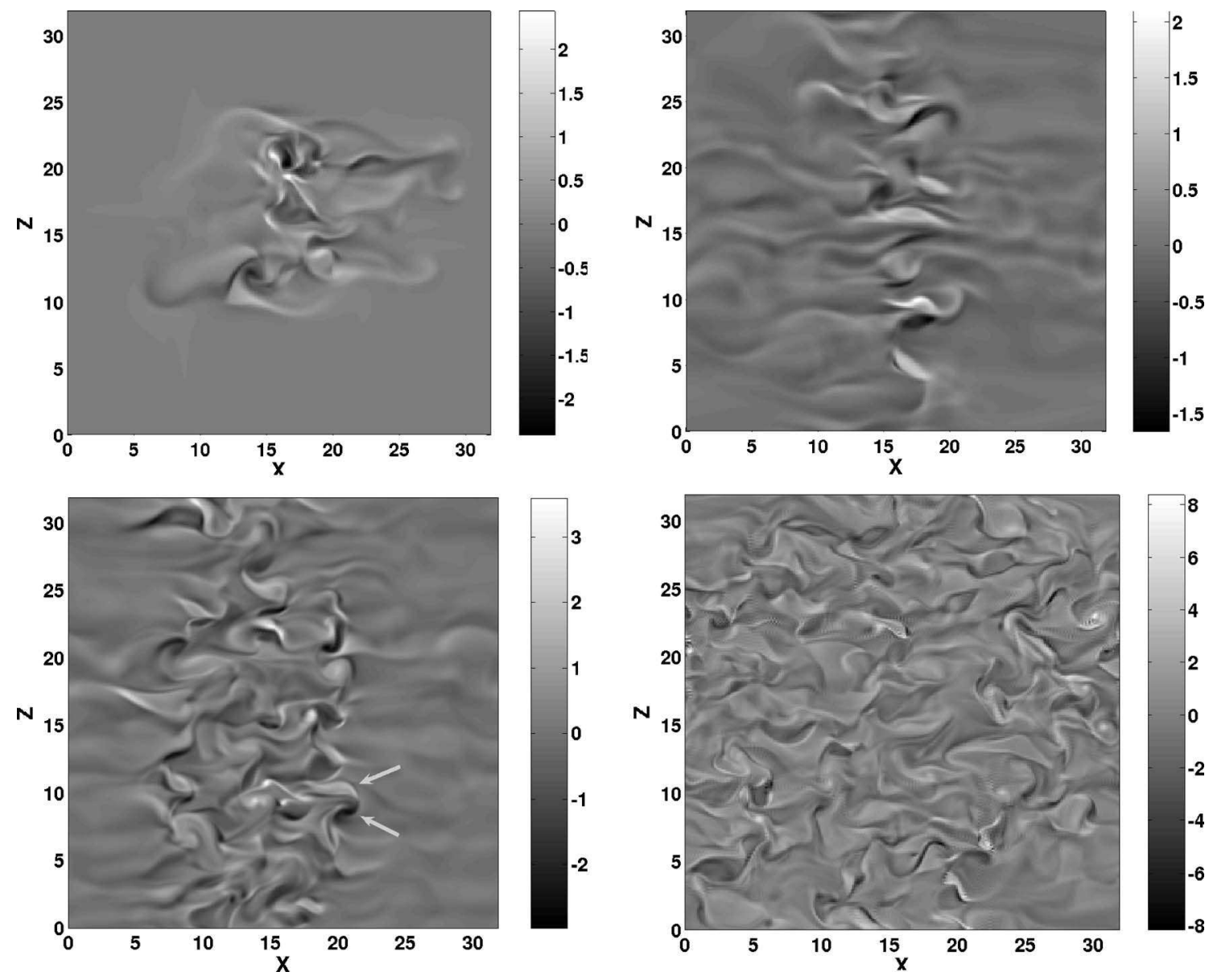

FIG. 1. Growth of a turbulent spot with $R=200$ in a domain with $L_{x} \times L_{z}=32 \times 32$. Wall-normal vorticity $\Delta_{2} \Psi_{0}$ in gray levels. From left to right and top to bottom: $t=20,70,156,210$.

more interestingly, the crescent vortices present in the front propagating to the right (in the streamwise direction or roughly in the oblique direction) are of the first kind, as is the one shown in Fig. 3, while the other kind populates the front propagating to the left. This observation is explained later on. The generation mechanism of these vortices is the subject of a forthcoming paper.

\section{THE SPREADING OF THE TURBULENT SPOT}

The numerical simulations of the model have been used to identify elementary processes involved in the spreading mechanism. The dipoles $\left(U_{0}, W_{0}\right)$ carry, during their propagation, the perturbation components $\left(U_{1}, V_{1}, W_{1}, \ldots\right)$ in the $(x, z)$ plane. This spreading mechanism has two points to be examined. The first concerns the origin of the motion of the dipoles while the second deals with the consequence of such motion.

Elements of proof for both points can be given by studying the contribution of the advective term $U_{0} \partial_{x} f+W_{0} \partial_{z} f$ in the governing equation of $f$, where the quantity $f$ can be the wall-normal vorticities $\Delta_{2} \Psi_{0}, \Delta_{2} \Psi_{1}$, or the velocity $V_{1}$, or the streamwise vorticity, etc. However, since this method produces data sets requiring a lot of posttreatment, it will be used only in Sec. III A to study the origin of the motion of the dipoles $\left(U_{0}, W_{0}\right)$ (first point). The entrainment of the per- turbation components by these dipoles (second point) is investigated using a model in Sec. III B.

\section{A. Origin of the dipole motion $\left(U_{0}, W_{0}\right)$}

Let us consider the governing equation for the streamfunction $\Psi_{0}$ of the drift flow $\left(U_{0}, W_{0}\right)$, given in the Appendix, which can be rewritten as

$$
\left[\partial_{t}-R^{-1}\left(\Delta_{2}-\gamma_{0}\right)\right] \Delta_{2} \Psi_{0}=J_{0}+J_{1}+J_{2}+J_{3}+J_{4}+J_{5},
$$

where the wall-normal vorticity of the flow $\left(U_{0}, W_{0}\right)$ is $\partial_{x} W_{0}-\partial_{z} U_{0}=\Delta_{2} \Psi_{0}$. On the right hand side (rhs) we have

$$
\begin{aligned}
& J_{0}=-\alpha_{1}\left(U_{0} \partial_{x} \Delta_{2} \Psi_{0}+W_{0} \partial_{z} \Delta_{2} \Psi_{0}\right), \\
& J_{1}=-a_{1}\left(U_{b}+\frac{\alpha_{2}}{a_{1}} U_{1}\right) \partial_{x} \Delta_{2} \Psi_{1}, \\
& J_{2}=-\alpha_{2}\left(\beta^{\prime}+\beta\right) V_{1} \Delta_{2} \Psi_{1}, \\
& J_{3}=\alpha_{2} \frac{3}{2}\left(U_{1} \partial_{z} \Delta_{2} \Phi_{1}-W_{1} \partial_{x} \Delta_{2} \Phi_{1}\right), \\
& J_{4}=-\alpha_{2} W_{1} \partial_{z} \Delta_{2} \Psi_{1}, \\
& J_{5}=a_{1} \frac{3}{2} U_{b} \partial_{z} \Delta_{2} \Phi_{1} .
\end{aligned}
$$



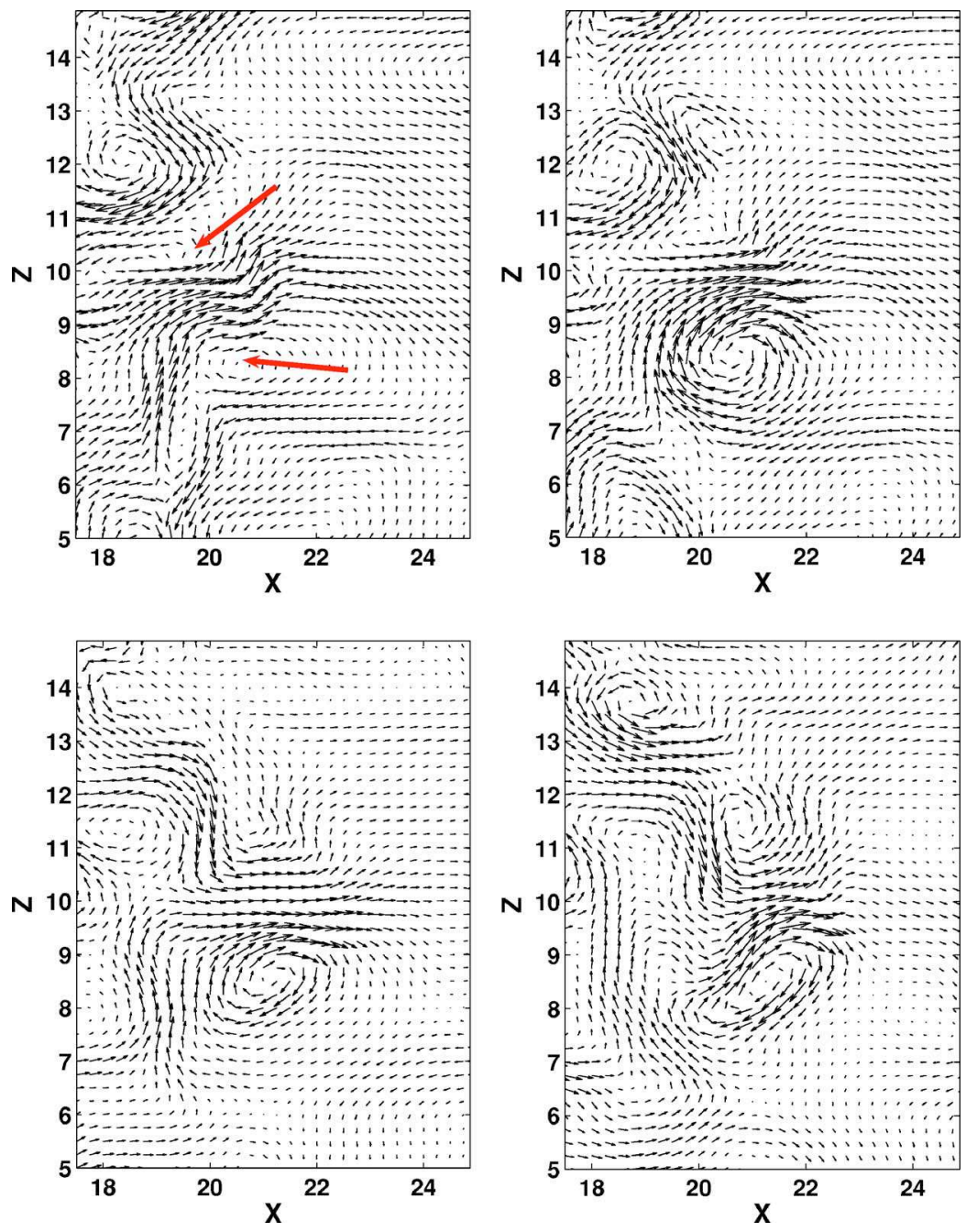

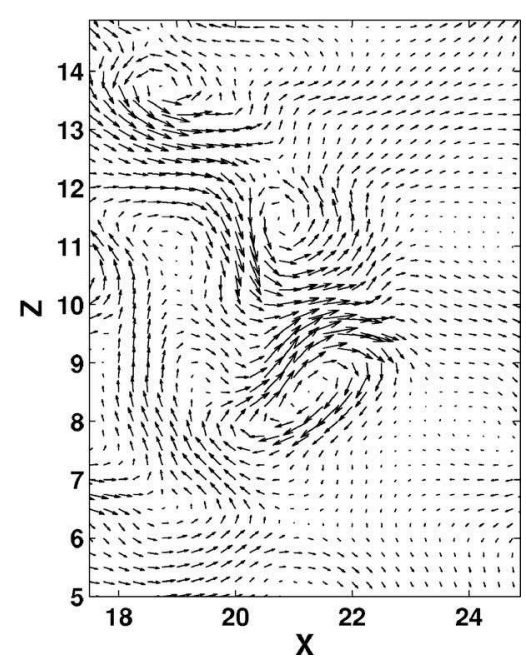

FIG. 2. The flow $\left(U_{0}, W_{0}\right)$ represented by arrows. From left to right and top to bottom: $t=154,156,160$, and 162.
The vorticity $\Delta_{2} \Psi_{0}$ results from the projection over $R_{0}(y)$ of the three-dimensional wall-normal vorticity $\partial_{x} w-\partial_{z} u$. In the same way, both terms $J_{0}$ and $J_{1}$ come from the projection over $R_{0}(y)$ of the term $u \partial_{x}\left(\partial_{x} w-\partial_{z} u\right)$ in the equation governing the vorticity $\partial_{x} w-\partial_{z} u$. The projection over $R_{1}(y)$ of $\partial_{x} w$ $-\partial_{z} u$ gives the vorticity $\Delta_{2} \Psi_{1}=\partial_{x} W_{1}-\partial_{z} U_{1}$.

Note that by setting the flow components $V_{1}, W_{1}$, and $U_{1}$ to zero, all the terms in the rhs of Eq. (4) vanish except $J_{0}$.
Hence, the equation governing the drift flow reduces to the two-dimensional Navier-Stokes equation, with an additional viscous damping $R^{-1} \gamma_{0}$ induced by the friction of this flow on the plates. The term $J_{0}$ represents the advection of the two-dimensional flow $\left(U_{0}, W_{0}\right) R_{0}$ for its own vorticity $\Delta_{2} \Psi_{0} R_{0}$.

The second term $J_{1}$ accounts for the generation of $\Delta_{2} \Psi_{0} R_{0}$ through the shearing of the vorticity $\Delta_{2} \Psi_{1} R_{1}$ by the
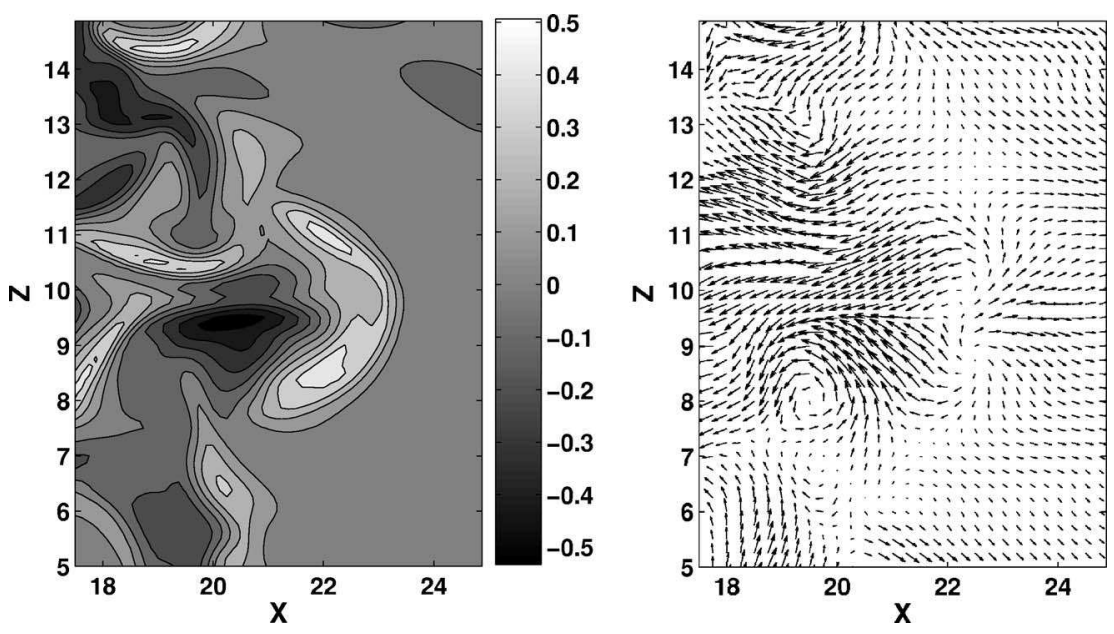

FIG. 3. The isocontours of $V_{1}$ (left) and the flow field $\left(U_{1}, W_{1}\right)$ (right) at $t=156$. This flow distribution represents a crescent vortex. 

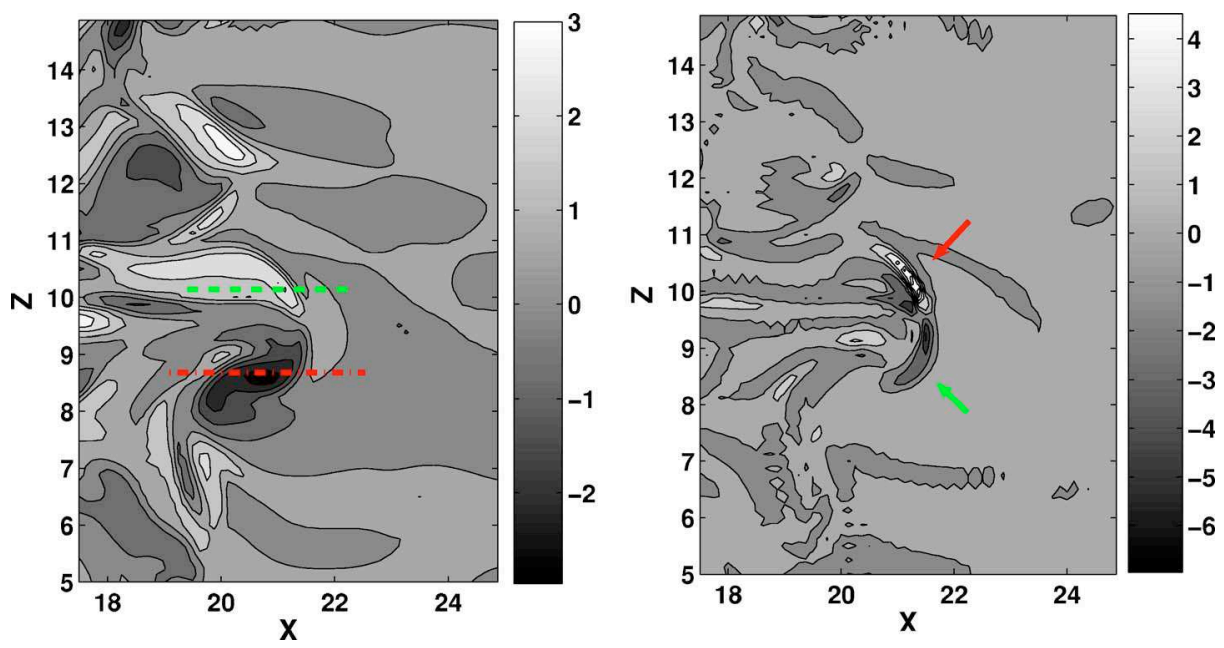

FIG. 4. (Color online) Left: Distribution of the wall-normal vorticity $\Delta_{2} \Psi_{0}$ at $t=156$. The two patches of $\Delta_{2} \Psi_{0}$ with positive and negative values correspond to the dipole depicted in Fig. 2. Right: The spatial distribution of $J_{0}$ presents two patches of positive and negative values in front of $\Delta_{2} \Psi_{0}$.

velocity component $u^{\prime}=U(y)+U_{1} R_{1}(y)$. In regions where $U_{1}$ is negative, $U_{1} R_{1}$ represents a correction to the base flow so that this $u^{\prime}$ has locally an $\mathbf{S}$ shape, similar to the mean profile of the turbulent plane Couette flow. ${ }^{16}$ In the following, the scalar $\tilde{U}_{b}=U_{b}+\alpha_{2} / a_{1} U_{1}$ as well as $u^{\prime}$ are termed the corrected base flow. Note finally that the lift-up effect is represented by the term $J_{5}$. Further interpretations of the terms in the rhs will be introduced on demand to analyze their different roles.

Consider now the dipole depicted at successive times in Fig. 2. Its wall-normal vorticity $\Delta_{2} \Psi_{0}$ consists of two adjacent patches with opposite signs as shown in Fig. 4 (left). In front of each one, there is a same-signed patch of $J_{0}$, given in the right panel of this figure. This distribution of $J_{0}$ is reminiscent of the distribution of the nonlinear term, in the twodimensional Navier-Stokes equation, in front of a propagating dipole. Hence, the origin of the motion of the considered dipole would be the self-advection of the flow $\left(U_{0}, W_{0}\right)$ if $J_{0}$ is preponderant over the other terms in the rhs of Eq. (4).
This is indeed the case as attested to by Fig. 5, where we plotted $\Delta_{2} \Psi_{0}$ between two successive instants as a function of $x$ along the streamwise the red (dash-dotted) line (for $z$ $\sim 9$ ) and the green (dashed) line (for $z \sim 10$ ) in Fig. 4 together with the whole rhs of Eq. (4). The term $J_{0}$ represents the largest contribution to this rhs, which is negative (positive) in front of the negative (positive) patch of $\Delta_{2} \Psi_{0}$ so that this vorticity propagates to the right. From a physical point of view, this propagation can be explained as the effect of the induced velocity of one vortex on the other in accordance to the Biot-Savart law.

In the following, we study the contributions, albeit weaker, of the other terms. Such study will give a clear picture of the roles of these terms.

First, the term $J_{1}$ acts against the propagation of the dipole by damping the vorticity $\Delta_{2} \Psi_{0}$. Indeed, this term is positive (negative) where $\Delta_{2} \Psi_{0}$ is negative (positive), as shown in Fig. 5. The origin of such behavior is as follows. Once the streaks $U_{0}$ are regenerated by the streamwise vor-
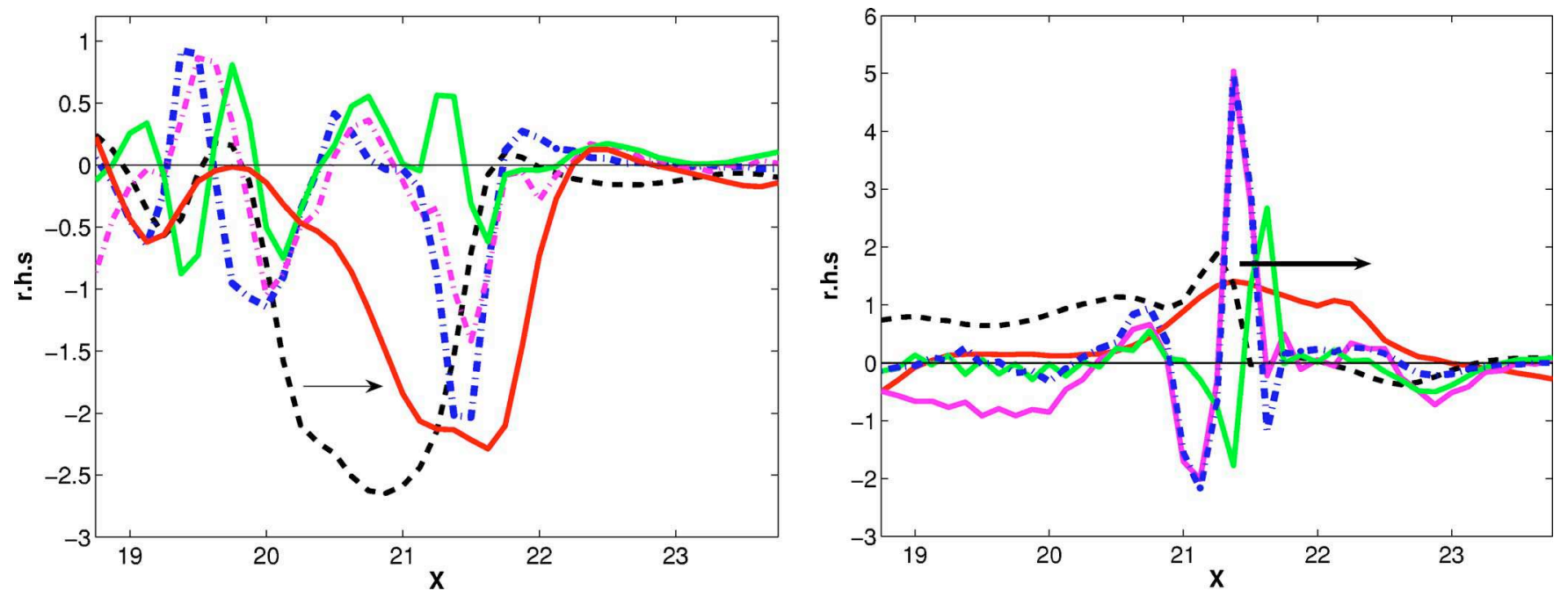

FIG. 5. (Color online) Different quantities as functions of coordinate $x$ along the dash-dotted red line (left panel) and dashed green line (right panel) in Fig. 4. The values of $\Delta_{2} \Psi_{0}$ at $t=156$ (in black dashed-line) and at $t=162$ (in solid red line). The total rhs of Eq. (4) in dashed magenta. $J_{0}$ in dash-dotted blue line and $J_{1}$ in solid green line. The arrows indicate the sense of propagation. 
tices, they are sheared by the base flow (linear transport) and induce the velocity component $U_{1}$ according to $\partial_{t} U_{1}$ $\sim-a_{1} U_{\mathrm{b}} \partial_{x} U_{0}$.

Within the formulation of the equations in terms of stream functions, this generation mechanism is represented by the linear term $-a_{1} U_{b} \partial_{x} \Delta_{2} \Psi_{0}$ in the equation governing $\Delta_{2} \Psi_{1}$ [(A5)]. Hence, the distribution of the induced vorticity $\Delta_{2} \Psi_{1}$ through the shearing of the vorticity $\Delta_{2} \Psi_{0}$ by the base flow is roughly given by $\Delta_{2} \Psi_{1} \sim-a_{1} U_{b} \partial_{x} \Delta_{2} \Psi_{0}$ (with $U_{b}=1$ ). It follows that

$$
J_{1}=-a_{1} \tilde{U}_{b} \partial_{x} \Delta_{2} \Psi_{1} \sim \tilde{U}_{b} \partial_{x x} \Delta_{2} \Psi_{0}
$$

showing that $J_{1}$ behaves like a dissipative term (with $\widetilde{U}_{b}$ $\geqslant 0)$.

Consider then the term $J_{2}$, which involves the quantities $V_{1}$ and $\Delta_{2} \Psi_{1}$. On the right of both patches of positive and negative values of $\Delta_{2} \Psi_{0}$, the velocity $V_{1}$ remains positive (crescent contour) whereas $\Delta_{2} \Psi_{1}\left(\sim-U_{b} \partial_{x} \Delta_{2} \Psi_{0}\right)$ changes its sign. It follows that in front of the dipole, $J_{2}$ and $J_{0}$ have opposite-signed distributions, thus the former term acts against the progression of the dipole.

The remaining terms $J_{3}, J_{4}$, and $J_{5}$ are now considered. The head of the crescent vortex is a spanwise vortex, where the flow $\left(U_{1}, W_{1}\right)$ is dominated by $\left|U_{1}\right| \gg\left|W_{1}\right|$ and $U_{1} \leqslant 0$ (although $U_{1} \sim 0$ in the present case). Hence, lumping the three terms and neglecting the contribution of $W_{1}$ yields

$$
J_{3}+J_{4}+J_{5} \approx a_{1} \frac{3}{2}\left(U_{b}+\frac{\alpha_{2}}{a_{1}} U_{1}\right) \partial_{z} \Delta_{2} \Phi_{1},
$$

which accounts for the lift-up effect, i.e., the extraction of the energy from the corrected base flow $\left(U_{b}+\alpha_{2} / a_{1} U_{1}\right)$ by the wall-normal velocity $V_{1}$. Therefore, the quantity $J_{3}+J_{4}+J_{5}$ is a source term for the streaks and thus for their wall-normal vorticity $\Delta_{2} \Psi_{0}$.

As a conclusion, by analyzing the different terms on the rhs of Eq. (4), this short study shows that (i) the dominant term is $J_{0}$ and (ii) the distributions of the different remaining terms can be determined since the involved quantities $\left(U_{1}, U_{0}, V_{1}, \ldots\right)$ are correlated through the cycle of selfsustained mechanisms for wall-bounded turbulence.

The propagation of the vortices $\left(U_{0}, W_{0}\right)$ is hence due to their self-advection (the term $J_{0}$ ). While some of these terms enhance the propagation, such as $J_{3}+J_{4}+J_{5}$, which intensifies the vorticity of the dipole, other terms act against this propagation by either weakening this vorticity, such as $J_{1}$, or by damping the contribution of $J_{0}$, such as $J_{2}$.

Note, however, that the preponderance of one term over the others is not permanent. For example, it is clear that during the linear growth of the vorticity $\Delta_{2} \Psi_{0}$ by the lift-up effect, the nonlinear contribution of $J_{0}$ is negligible compared to that of $J_{5}$. In other words, arguing that a term dominates another one necessitates the explicit reference to which mechanism in the cycle is occurring. In this study, we were only concerned about the origin of the propagation of the dipoles once they are generated and about the roles of the different terms on the rhs of Eq. (4) in this propagation.
In the following, the consequence of the motion of the dipoles on the other perturbation components is studied using a set of one-dimensional partial differential equations.

\section{B. Entrainment of the perturbations: An illustrative model}

A simple model is now derived to illustrate the entrainment of the perturbations in the $x$ direction through the motion of the dipoles $\left(U_{0}, W_{0}\right)$. The $z$ dependence of the perturbations is frozen on some Fourier modes. The symmetries of the problem are then used to simplify the expansions of the fields by choosing a set of functions satisfying a particular symmetry. This choice is driven by the fact that the wallnormal vorticity of a dipole propagating in the $x$ direction is odd in $z$.

Hence, the fields $\Psi_{0}, \Psi_{1}$, and $\Phi_{1}$ for such a solution have these Fourier expansions:

$$
\begin{aligned}
& \Psi_{0}=\sum_{n \geqslant 1} A_{n}(x, t) \sin (n \theta z), \\
& \Psi_{1}=\sum_{n \geqslant 1} B_{n}(x, t) \sin (n \theta z), \\
& \Phi_{1}=\sum_{n \geqslant 0} C_{n}(x, t) \cos (n \theta z),
\end{aligned}
$$

where $\theta=2 \pi / L_{z}$ is the spanwise fundamental.

A stream-function of a dipole $\left(U_{0}, W_{0}\right)$ can be represented by the first mode $\Psi_{0}(x, z, t)=A_{1}(x, t) \sin (\theta z)$. However, due to the $z$ periodicity, this dipole cannot propagate in the $x$ direction. To remedy this, it is sufficient to include the second harmonic in this expansion, $A_{2}(x, t) \sin (2 \theta z)$. The superposition of these two modes yields a modulated array of vortices in the spanwise direction.

Then for the stream-function $\Psi_{1}$, we have to include the first two modes, since the vorticity $\Delta_{2} \Psi_{1}$ is linearly generated from $\Delta_{2} \Psi_{0}$ through the linear term $-a_{1} U_{b} \partial_{x} \Delta_{2} \Psi_{0}$ [Eq. (A5)].

Last, the expansion of the potential velocity $\Phi_{1}$, which is related to the wall-normal velocity $V_{1}$ of the vortices, is truncated. The nonlinear interactions of the in-plane $(x, z)$ flow components induce this $V_{1}$ through the terms $-U_{1} \partial_{z} W_{0}$ and $W_{0} \partial_{z} U_{1}$. Accordingly, we have to keep the Fourier modes generated by these terms with $U_{1}=-\partial_{z} \Psi_{1}$ and $W_{0}=\partial_{x} \Psi_{0}$. With the retained modes for $\Psi_{0}$ and $\Psi_{1}$, these modes are 1, $\cos (\theta z), \cos (2 \theta z)$, and $\cos (3 \theta z)$. Finally, the expansions read

$$
\begin{aligned}
\Psi_{0}= & A_{1}(x, t) \sin (\theta z)+A_{2}(x, t) \sin (2 \theta z), \\
\Psi_{1}= & B_{1}(x, t) \sin (\theta z)+B_{2}(x, t) \sin (2 \theta z), \\
\Phi_{1}= & C_{0}(x, t)+C_{1}(x, t) \cos (\theta z)+C_{2}(x, t) \cos (2 \theta z) \\
& +C_{3}(x, t) \cos (3 \theta z) .
\end{aligned}
$$



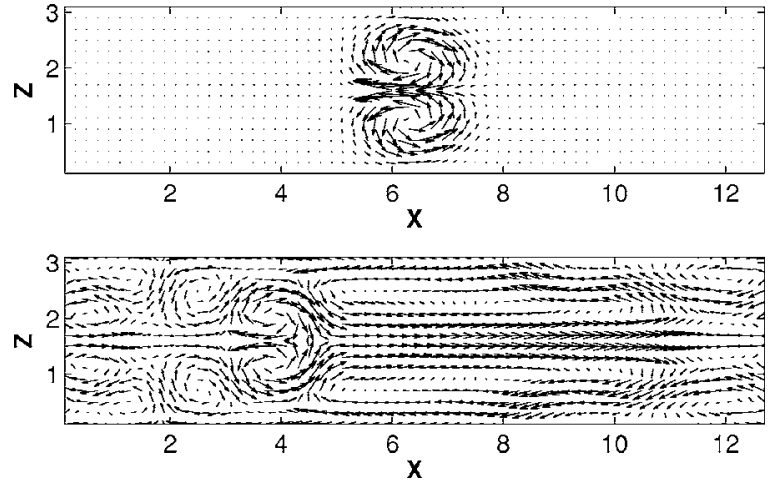

FIG. 6. The propagating dipole depicted by the flow $\left(U_{0}, W_{0}\right)$ at $t=0.1$ (top) and $t=5$ (bottom). $R=200$.

\section{Numerical results}

The numerical integration of the illustrative model can be easily done using the code already developed for the noslip model. Between two successive time steps, all the Fourier modes of the three fields are set to zero except the retained modes in the expansions above.

As an initial condition, we take $A_{1}(x, 0)$ $=-4 / 5 \pi^{2} e^{-2\left(x-x_{\mathrm{C}}\right)^{2}}, \quad A_{2}(x, 0)=-\frac{1}{2} A_{1}(x, 0), \quad C_{0}=C_{1}=C_{2}=C_{3}$ $=0, \quad B_{1}(x, 0)=-4 / 9 \sqrt{\sqrt{3 \pi}} e^{-\left(x-x_{C}\right)^{2} / 6}, \quad$ and $\quad B_{2}(x, 0)=-\frac{1}{2} B_{1}$ $(x, 0)$. The streamwise length of the computational domain is $L_{x}=12.8$ with $x_{C}=L_{x} / 2$ and the Reynolds number is $R=200$. Choosing $\theta=2 \pi / L_{z}$ with $L_{z}=3.2$ yields a streak $U_{0}$ with a spanwise width of about $\approx L_{z} / 2=1.6$. With the retained value of $\theta$, we have $U_{0}(x, z, 0) \approx O(1)$ and $U_{1}(x, z, 0) \approx O(1)$.

First, the dipole $\left(U_{0}, W_{0}\right)$ depicted in Fig. 6 is propagating to the left, as could be inferred from the sense of rotation of both vortices. This propagation is also tracked by its vorticity $\Delta_{2} \Psi_{0}$ as shown in Fig. 7 . Second, during its propagation, this dipole carries the other flow components. Indeed, Fig. 8 illustrates the generation and the entrainment of a crescent vortex to the left, depicted by the wall-normal velocity $V_{1}$. The corresponding flow field $\left(U_{1}, W_{1}\right)$ is given in Fig. 9. The legs of this crescent are two streamwise vortices characterized by two patches of streamwise vorticity $\omega_{x}$ $=\beta W_{1}-\partial_{z} V_{1}$, as shown in Fig. 10. During their propagation to the left, they regenerate the streaks and produce positive Reynolds stress $-U_{0} V_{1}$ : positive (negative) patches of $V_{1}$ cor-
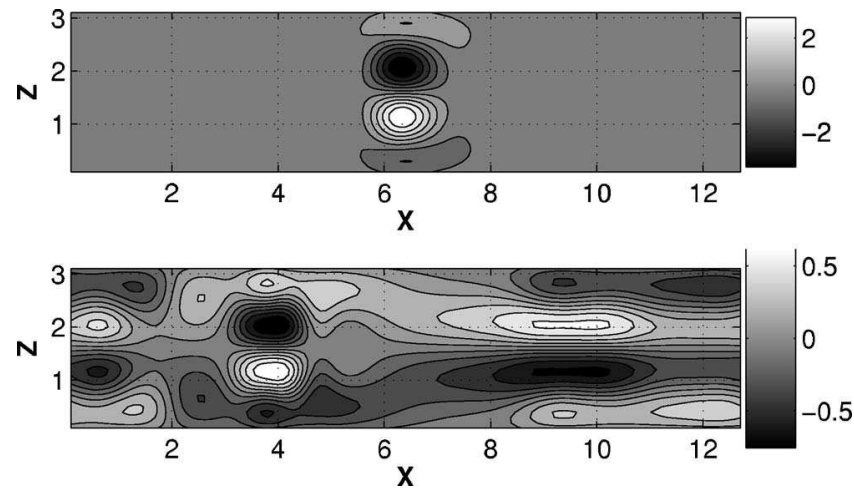

FIG. 7. The wall-normal vorticity $\Delta_{2} \Psi_{0}$ at $t=0.1$ (top) and $t=5$ (bottom).
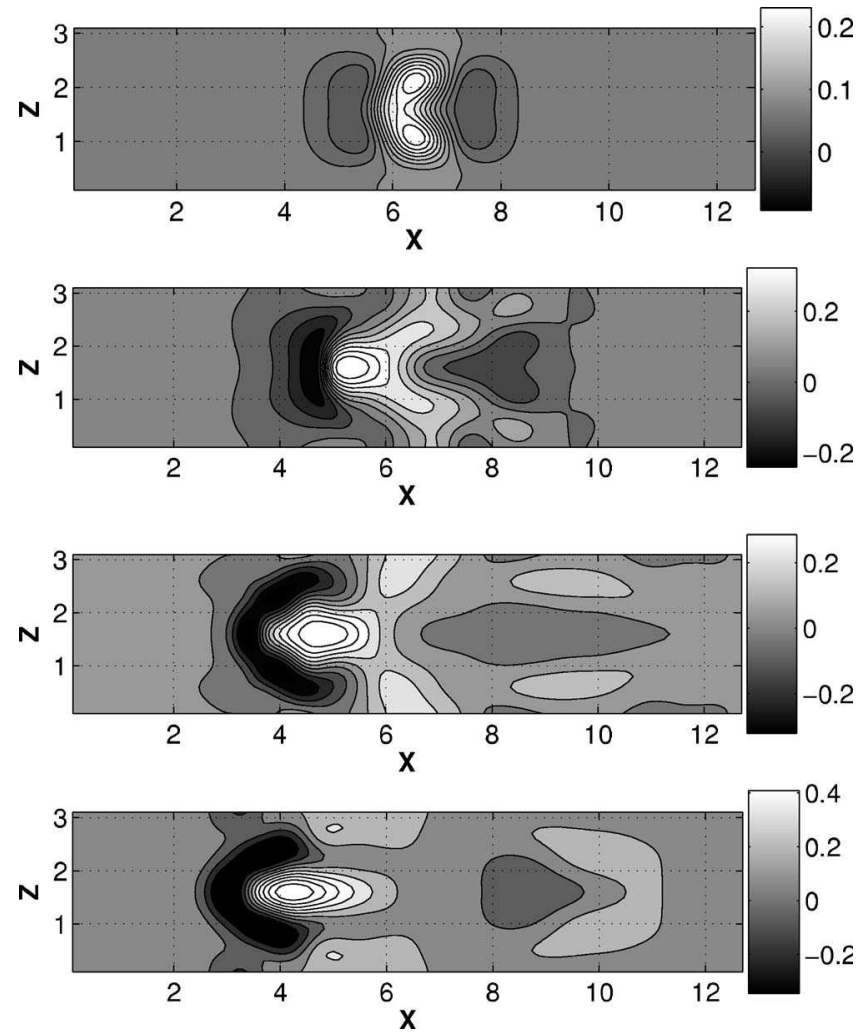

FIG. 8. Generation and entrainment of a crescent vortex, depicted by its vertical velocity $V_{1}$. From top to bottom, $t=0.1,1,3$, and 5 . The flow field $\left(U_{1}, W_{1}\right)$ is given below in Fig. 9.

respond to the negative (positive) regions of $U_{0}$. Hence during its motion, the dipole $\left(U_{0}, W_{0}\right)$ carries the crescent vortex, which continues to regenerate it through the lift-up effect.

Afterwards, the wall-normal vorticity $\Delta_{2} \Psi_{0}$ associated with these streaks is sheared by the base flow and a vorticity $\Delta_{2} \Psi_{1}$ is induced. Its distribution is roughly given by $\Delta_{2} \Psi_{1}$ $\sim-a_{1} U_{b} \partial_{x} \Delta_{2} \Psi_{0}$, as shown in Fig. 10 by the arrows. In turn, this vorticity damps the progression of the dipole (the term $\left.J_{1}\right)$.

Finally we have stated in Sec. II that, for a given spot, each front was populated by a particular kind of crescent vortex. The reason behind this distribution is simple. Depending on the sense of rotation of the dipole $\left(U_{0}, W_{0}\right)$, a spanwise vortex is deformed by this dipole and gives a crescent vortex of the first or the second kind. If it is of the first kind (such as the one depicted in Fig. 8), it is advected to the left, whereas if it is of the second kind, it is advected to the right (such as the one depicted in Fig. 3).

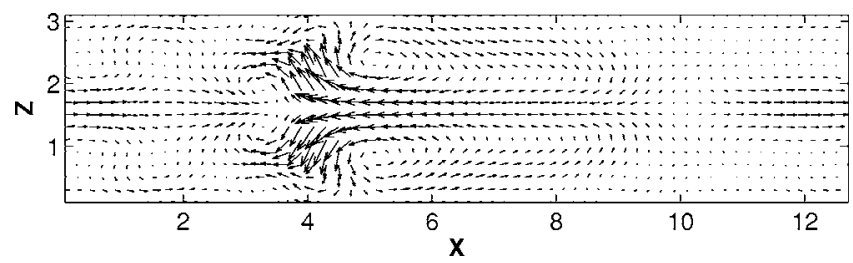

FIG. 9. The flow field $\left(U_{1}, W_{1}\right)$ at $t=5$. This flow field together with the corresponding $V_{1}$ represent a crescent vortex. 

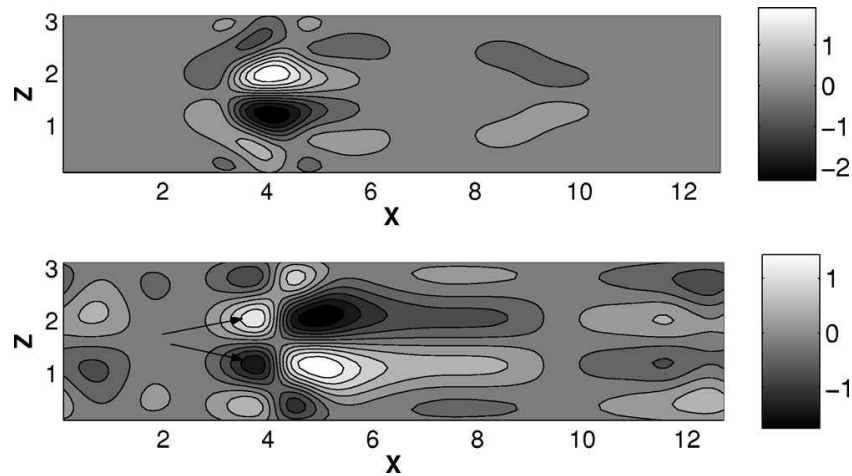

FIG. 10. Top: The streamwise vorticity $\omega_{x}$. Bottom: The wall-normal vorticity $\Delta_{2} \Psi_{1}$ at $t=5$.

As a conclusion, this model illustrates well the entrainment of the flow quantities by a propagating dipole $\left(U_{0}, W_{0}\right)$. The interactions between the flow components encountered in the preceding section, such as the lift-up effect, are well captured by this model. Its natural limitation is that the dipole is allowed to propagate only in the $x$ direction, since its $z$ dependence is frozen.

\section{DISCUSSION AND CONCLUSION}

In this paper, the spreading of a turbulent spot was investigated. The contamination of the laminar domain by the turbulent domain is a consequence of the motion in the horizontal plane of wall-normal vortices spanning all of the gap between the two plates.

First, we have shown that the dynamic of these dipoles is governed by their self-advection. Second, during their motion, these dipoles carry the other perturbation components and continue to interact with them. For instance, the carried crescent vortex continues to regenerate the streaks by the lift-up mechanism. As a consequence of this entrainment by the dipoles, the front propagating to the right is populated by crescent vortices of the first kind, whereas the one propagating to the left is populated by crescent vortices of the second kind. The core of the spot, however, is filled with both kinds, as shown in Fig. 11.

Furthermore, our aim is to present the elementary building block of the spreading mechanism, which is the motion of the dipoles. For this reason, we did not study the interactions of these blocks, for instance when two dipoles moving in opposite directions encounter one another and then propagate in the spanwise direction, as shown in Fig. 11. Moreover, depending on the sense of their rotation, the dipoles propagate in the $(x, z)$ plane toward the $x$ direction but also toward the oblique direction, since they are not all symmetric in $z$. Figure 2 (for $t=156$ ) gives an example of such a dipole (for $z \geqslant 11, x \leqslant 21$ ). Its vorticity is shown in Fig. 4.

We have seen in Ref. 16 that on the outskirts of the turbulent spot, there is a quadrupolar large-scale flow denoted by $\left(\overline{U_{0}}, \overline{W_{0}}\right)$. Some possible consequences of this flow on the spreading of the spot can be provided by the present study. The large-scale streamwise inflow $\overline{U_{0}}$ could hinder this spreading since it pushes the small-scale dipoles $\left(U_{0}, W_{0}\right)$

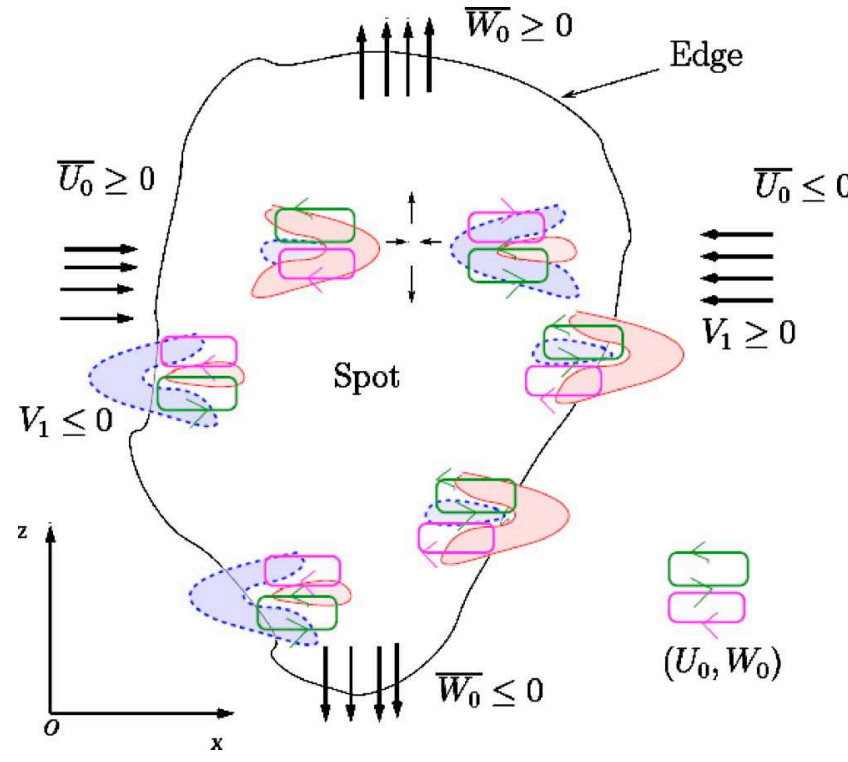

FIG. 11. (Color online) General view of the spreading mechanism, with the role of the large-scale flow outside the spot. The outflow contributes in the spreading of the spot in the spanwise direction, whereas the inflow acts against the progression of the small-scale dipoles $\left(U_{0}, W_{0}\right)$ in the $x$ direction. Red-solid (blue-dashed) contours indicate regions of positive (negative) values (enhanced online).

toward the core of the spot, thus acting against their progression in the streamwise direction, as illustrated in Fig. 11.

In contrast, the large-scale outflow $\overline{W_{0}}$ contributes to the spreading of the spot in the spanwise direction by advecting the perturbations in the core of the spot outwards. This outflow $\overline{W_{0}}$ acting in both spanwise directions would explain the spot-splitting phenomenon, occurring at Reynolds numbers close to the transitional and observed in experimental studies (see, e.g., Ref. 1) and in other shear flows experiencing a transition to turbulence by nucleation of spots, such as plane Poiseuille flow. ${ }^{2}$ Its existence in these shear flows was discussed in Ref. 16.

Despite its limited cross-stream resolution, our model for PCF gives valuable hints about the spreading mechanism of turbulent spots. First, the presented mechanism still holds when we increase the wall-normal resolution. Second, the models derived in Ref. 11 for PCF with free-slip boundary conditions on the plates for different resolutions in $y$ exhibit the same spreading mechanism as in the no-slip case. Third, evidence of the presence of the dipoles on the front can be obtained from laboratory experiments by measuring the wallnormal vorticity either at the midplane or by averaging it over the gap. The experimental investigation of Schröder and Kompenhaus $^{17}$ is an example of such studies but in other wall flow of more practical interest than PCF. Their Figs. 6 and 13 describe the result of an ensemble average of the wall-normal vorticity of spots in boundary-layer flow and show small adjacent regions of opposite-signed vorticity.

Moreover, postprocessing techniques have to be developed to quantify the contribution of the proposed mechanism to the spreading of the turbulent spot. The attributes of the vortical structures at the boundary, such as length scale and drift velocity, as well as their variation with the Reynolds 
number, could be investigated by these techniques, rather than using instantaneous flow visualization.

Clearly, further studies investigating the internal structures of turbulent spots and especially the substructures on the edge of the spot are needed to conclusively comment on the spreading mechanism presented in this paper and its occurrence in a real turbulent spot. The experimental works of Perry $^{18}$ and of Sankaran et al., ${ }^{19,20}$ who investigated the structure of a spot in boundary layer flow, as well as the numerical investigation of Singer ${ }^{21}$ and the recent experiments of Makita and Nishizawa ${ }^{22}$ are examples of such studies.

Finally, the present discussion of the spreading mechanism and the flow patterns at the boundaries could be a stepping stone for a quantitative estimation and derivation of the front propagation speed $v_{\mathrm{fr}}$. The formula $v_{\mathrm{fr}} \propto \Gamma / 2 \pi r$, where $\Gamma$ is the recirculation of the vortices $\left(U_{0}, W_{0}\right)$ and $r$ is related to the streak spacing, could be a good starting point.

\section{APPENDIX: EQUATIONS OF THE MODEL}

Inserting the expansions (1)-(3) in the Navier-Stokes and continuity equations and following the Galerkin method prescription yields the governing equations of the amplitudes $U_{0}, W_{0}, U_{1}, V_{1}$, and $W_{1}$. For instance, the continuity equation gives the following relations:

$$
\partial_{x} U_{0}+\partial_{z} W_{0}=0, \quad \partial_{x} U_{1}+\partial_{z} W_{1}=\beta V_{1},
$$

with $\beta=\sqrt{3}$. Then, defining the appropriate stream-functions $\Psi_{0}$ and $\Psi_{1}$ and the velocity potential $\Phi_{1}$ as follows:

$$
U_{0}=-\partial_{z} \Psi_{0}, \quad W_{0}=\partial_{x} \Psi_{0},
$$

and

$$
U_{1}=\partial_{x} \Phi_{1}-\partial_{z} \Psi_{1}, \quad W_{1}=\partial_{z} \Phi_{1}+\partial_{x} \Psi_{1}, \quad \beta V_{1}=\Delta_{2} \Phi_{1},
$$

and using the governing equations of the velocity amplitudes $\left(U_{0}, W_{0}, U_{1}, W_{1}\right.$, and $\left.V_{1}\right)$ together with the continuity equations (A1) yield three partial derivative equations governing the evolution of the fields $\Psi_{0}, \Psi_{1}$, and $\Phi_{1}$, which constitute our no-slip model. These equations are

$$
\begin{aligned}
{\left[\partial_{t}-R^{-1}\left(\Delta_{2}-\gamma_{0}\right)\right] \Delta_{2} \Psi_{0}=} & \left(\partial_{z} N_{U_{0}}-\partial_{x} N_{W_{0}}\right)+a_{1}\left(\frac{3}{2} U_{b} \partial_{z} \Delta_{2} \Phi_{1}\right. \\
& \left.-U_{b} \partial_{x} \Delta_{2} \Psi_{1}\right), \\
{\left[\partial_{t}-R^{-1}\left(\Delta_{2}-\gamma_{1}\right)\right] \Delta_{2} \Psi_{1}=} & \left(\partial_{z} N_{U_{1}}-\partial_{x} N_{W_{1}}\right)-a_{1} U_{b} \partial_{x} \Delta_{2} \Psi_{0},
\end{aligned}
$$

$$
\begin{aligned}
& {\left[\partial_{t}-R^{-1}\left(\Delta_{2}-\beta^{2}\right)\right]\left(\Delta_{2}-\beta^{2}\right) \Delta_{2} \Phi_{1}} \\
& \quad=\beta^{2}\left(\partial_{x} N_{U_{1}}+\partial_{z} N_{W_{1}}\right)+\frac{45}{2} R^{-1} \Delta_{2} \Phi_{1}-\beta \Delta_{2} N_{V_{1}},
\end{aligned}
$$

where $R$ is the Reynolds number and $\Delta_{2}$ is the twodimensional Laplacian $\partial_{x x}+\partial_{z z}$. The nonlinear terms $N_{U_{0}}$, $N_{W_{0}}, N_{U_{1}}, N_{W_{1}}$, and $N_{V_{1}}$ as well as the values of the positive constants $\left(a_{1}, \gamma_{0 . . .}\right)$ can be found in Ref. 16 .

${ }^{1}$ O. Dauchot and F. Daviaud, "Finite amplitude perturbation and spots growth-mechanism in plane Couette flow," Phys. Fluids 7, 335 (1995).

${ }^{2}$ D. R. Carlson, S. E. Widnall, and M. F. Peeters, "A flow-visualization study of transition in plane Poiseuille flow," J. Fluid Mech. 21, 487 (1982).

${ }^{3}$ M. Gad-El-Hak, R. F. Blackwelder, and J. J. Riley, "On the growth of turbulent regions in laminar boundary layers," J. Fluid Mech. 110, 73 (1981).

${ }^{4}$ A. Lundbladh and A. V. Johansson, "Direct simulation of turbulent spots in plane Couette flow," J. Fluid Mech. 229, 499 (1991).

${ }^{5} \mathrm{~J}$. Schumacher and B. Eckhardt, "Evolution of turbulent spots in a parallel shear flow," Phys. Rev. E 63, 046307 (2001).

${ }^{6}$ L. Kleiser and T. A. Zang, "Numerical simulation of transition in wall bounded shear flows," Annu. Rev. Fluid Mech. 23, 495 (1991).

${ }^{7} \mathrm{~N}$. Tillmark and P. H. Alfredsson, "Experiments on transition in plane Couette flow," J. Fluid Mech. 235, 89 (1992).

${ }^{8} \mathrm{~N}$. Tillmark, "On the spreading mechanisms of a turbulent spot in plane Couette flow," Europhys. Lett. 32, 481 (1995).

${ }^{9}$ O. Dauchot and F. Daviaud, "Finite amplitude perturbation in plane Couette flow," Europhys. Lett. 28, 225 (1994).

${ }^{10}$ S. Bottin, O. Dauchot, F. Daviaud, and P. Manneville, "Experimental evidence of streamwise vortices as finite amplitude solutions in transitional plane Couette flow," Phys. Fluids 10, 2597 (1998).

${ }^{11} \mathrm{P}$. Manneville, "Spots and turbulent domains in a model of transitional plane Couette flow," Theor. Comput. Fluid Dyn. 18, 169 (2004).

${ }^{12}$ J. Mathew and A. Das, "Direct numerical simulations of spots," Curr. Sci. 79, 816 (2000).

${ }^{13}$ D. S. Henningson, P. Spalart, and J. Kim, "Numerical simulations of turbulent spots in plane Poiseuille and boundary-layer flow," Phys. Fluids 30, 2914 (1987)

${ }^{14}$ D. S. Henningson, "Wave growth and spreading of a turbulent spot in plane Poiseuille flow," Phys. Fluids A 1, 1876 (1989).

${ }^{15}$ F. Alavyoon, D. S. Henningson, and P. H. Alfredsson, "Turbulent spots in plane Poiseuille flow-flow visualization," Phys. Fluids 29, 1328 (1986).

${ }^{16}$ M. Lagha and P. Manneville, "Modeling of plane Couette flow. I. Large scale flow around turbulent spots," Phys. Fluids 19, 094105 (2007) (referred to as Part I).

${ }^{17}$ A. Schröder and J. Kompenhans, "Investigation of a turbulent spot using multi-plane stereo particle image velocimetry," Exp. Fluids 36, 82 (2004).

${ }^{18}$ A. E. Perry, T. T. Lim, and E. W. Teh, "A visual study of turbulent spots," J. Fluid Mech. 104, 387 (1981).

${ }^{19}$ R. Sankaran, M. Sokolov, and R. A. Antonia, "Substructures in a turbulent spot," J. Fluid Mech. 197, 389 (1988).

${ }^{20}$ R. Sankaran, R. A. Antonia, D. K. Bisset, and M. Sokolov, "Flow patterns and organization within a turbulent spot," Phys. Fluids A 3, 1560 (1991).

${ }^{21}$ B. A. Singer, "Characteristics of a young turbulent spot," Phys. Fluids 8, 509 (1996).

${ }^{22}$ H. Makita and A. Nishizawa, "Characteristics of internal vortical structures in a merged turbulent spot," J. Turbul. 2, 1 (2001). 